\title{
Prisms of Whiteness: A Critical Analysis of the Multicultural Competence of Pre-Service Teachers
}

\author{
Joshua B. L. Tolbert \\ Indiana University East \\ Indiana, U.S.A.
}

\begin{abstract}
This study endeavored to assess the cultural competency of pre-service teachers in a region of the United States with limited ethnic or linguistic diversity. To do this, the prior work of D'Andrea, Daniels, and Noonan (2003) was used to develop an updated survey instrument. Students at a small university in the Midwest were surveyed at multiple intervals during the course of their teacher education program, and their total responses $(n=190)$ are analyzed here. Findings for reliability and validity of the instrument are discussed, as well as general trends when comparing students by course enrollment, major, and degree status. Further discussion centers on the potential discovery of a partial measure of white fragility and how the results of the study may be enhanced by applying a lens of critical whiteness. Finally, implications are presented regarding how white fragility might be directly and deliberately incorporated into future efforts to measure and develop cultural competency in pre-service teachers.
\end{abstract}

Keywords: white fragility, cultural competency, multicultural education, critical whiteness.

\section{Introduction}

Ensuring that future K-12 educators are immersed in pedagogical research and practice which prioritizes cultural competence has been a consistent, if imperfect priority of teacher preparation programs. A frequently cited rationale for this emphasis on situating teaching in the context of a pluralistic society has been a shifting population, namely one in which students from historically marginalized or underrepresented groups represent ever-larger percentages of the U.S. school population (National Center for Educational Statistics, 2000; Warring, 2005). Despite the continued shift in population demographics, the K12 teaching profession remains predominantly white (Frankenberg, 2008; Warring, 2005); approximately $84 \%$ of full-time higher education faculty were also white (NCES, 2015, Sensoy \& DiAngelo, 2017). Given the general increases 
in cultural and linguistic diversity in the United States, in juxtaposition to the recent documented rise of hate crimes and white supremacist groups (Center for the Study of Hate and Extremism, 2018; Southern Poverty Law Center, 2019), cultural competency is a priority for all citizens and a vital attribute of educators.

\section{Teaching and Measuring Cultural Competency}

Although multicultural education and cultural competence have been established as important, different perspectives have existed on how to promote, measure, or contextualize these topics. Ladson-Billings (1994) promoted a culturally-relevant form of pedagogy that embraced the experiences and perspectives of all students, consciously remaining distinct from the assumptions of the dominant culture. Banks (1995) asserted that multicultural competence could be operationalized using three constructs: awareness, knowledge, and skills. The three aforementioned constructs have also been a staple in the field of counseling, where multicultural competence has been of similar importance. Sue, Arredondo, and McDavis (2002) specifically advocated for multicultural competence in counseling, developing and refining a tripartite model similar to what Banks described.

This tripartite model which incorporated awareness, knowledge, and skills has also served as the foundation for multiple instruments which endeavored to assess cultural competency in service-oriented fields like counseling, social work, and teacher education. The Multicultural Awareness, Knowledge, and Skills Survey (MAKSS) was developed by D'Andrea, Daniels, and Heck (1991) and eventually adapted into the teacher-oriented MAKSS-Form T ( $\mathrm{D}^{\prime}$ Andrea, Daniels, and Noonan, 2003). Spanierman et al. (2011) attempted to adapt the tripartite model into the Multicultural Teaching Competency Scale (MTCS), citing concerns that "no psychometrically viable scale assesses the multidimensional aspects depicted in the tripartite model of multicultural competence" (p. 443). Notably, Spanierman et al. (2011) were unable to produce a valid and reliable scale to measure awareness, and therefore presented the MTCS as a viable instrument with two factors: knowledge and skills. Prieto (2012) experienced a similar difficulty in developing the Multicultural Teaching Competencies Inventory (MTCI); only one item loaded above .40, and the entire Awareness subscale was consequently deleted. Jones and Walker (2017) similarly developed and tested the Multicultural Awareness, Skills, and Knowledge Survey (MASKS) with a sample of pre-service teachers enrolled at community colleges. The MASKS was notable for producing an awareness scale that did meet validation criteria.

The Awareness subscale has been the subject of a related trend involving comparatively low reliability in several previously published reports of prior research which utilized different versions of the MAKSS. As reported by Warring (2005), the 1991 version of the MAKSS produced a reliability coefficient of .75 for the Awareness subscale, as compared to the values for Knowledge (.90) and Skills (.96). This trend essentially held when D'Andrea, Daniels, and Noonan (2003) updated the MAKSS-Form $\mathrm{T}$ and computed a reliability coefficient of .73 for the Awareness subscale, in contrast to the coefficients for 
Knowledge (.86) and Skills (.93). Hall and Theriot (2016) experienced a similar pattern when using a version of the MAKSS as a pre- and post-assessment instrument with students majoring in social work. The Awareness subscale once again produced lower reliability coefficients (.46 pre/.58 post) than for Knowledge (.75 pre/.75 post) or Skills ( .85 pre/.92 post).

The tendency for different versions of the MAKSS to fall short of validation criteria on the Awareness subscale presented an enticing research question for the present study. This was one reason for the ultimate decision to develop an adaptation of the MAKSS-Form T, but another rationale came from other, earlier work from the primary researchers behind the MAKSS. D'Andrea and Daniels (1999) asserted that they had "conducted research that focused on the underpinnings of White racism over the last 16 years" (p. 93). Although this overt confrontation to white racism arguably did not manifest itself explicitly in the MAKSS, the intentions of the original researchers made the MAKSS uniquely suited to exploring multicultural competence in a largely white region of the Midwest, and in a historical era in which a rise in white hostility constituted an alarming social trend.

As noted by DiAngelo (2018), white people in the United States have historically been insulated from matters of race, while still benefiting from a system that privileges whiteness. Because white people in the U.S. have been largely insulated from racism or exempt from the expectation to define oneself in racial terms, white Americans may be ill-prepared for difficult dialogues about matters of race or culture (DiAngelo, 2018). The honest discourses and self-reflection ultimately required to develop cultural competence or promote social progress are rejected by many white people using tactics like silence or dismissal. DiAngelo (2018) categorized these tactics as white fragility, serving to "reinstate white equilibrium as they repel the challenge, return our racial comfort, and maintain our dominance within the racial hierarchy" (p. 2). Not unlike LadsonBillings (2004) and Banks (1995), DiAngelo has been striving to confront and disrupt narratives and presumptions that position whiteness as an unquestioned norm. Importantly, white fragility and related concepts like white silence (DiAngelo, 2012) or white innocence (Orozco \& Diaz, 2016) have flourished in the field of critical whiteness studies and have provided a more expansive vocabulary and a wider lens through which to view the relationship between whiteness and multicultural competence.

\section{Aims of the Study}

The research summarized here was largely rooted in the need to develop an additional measure of cultural competency as part of assessing and improving a teacher preparation program at a small university in the Midwest. In review of the existing literature described above, this analysis will also explore the following questions:

1. Can an adapted version of the MAKSS-Form $T$ result in valid and reliable subscales for Awareness, Knowledge, and Skills? 
2. Can the data collected from predominantly white students in this particular teacher preparation program be meaningfully discussed within the context of white fragility or related concepts from the field of critical whiteness studies?

\section{Development of the MAKSS-TEP}

The first version of the MAKSS obtained by the researchers was the MAKSSForm $\mathrm{T}$ attributed to D'Andrea, Daniels, and Heck, and understood to be derived from the version of the MAKSS used for counseling education (D'Andrea, Daniels, \& Heck, 1991). Since the original MAKSS-Form T included 60 items, a committee of faculty members at our university was consulted to streamline the total number of items and to update items in ways that would promote clarity or parsimony. Whenever possible, the original phrasing from the MAKSS-Form $\mathrm{T}$ was kept intact, for the sake of direct comparison of findings. It was decided that the instrument would use a four-item Likert-style scale consisting of "Strongly Disagree," "Disagree," "Agree," and "Strongly Agree." The choice not to include a "Neutral" or "No Opinion" option was deliberate, as it was deemed important to have a clearer measure of students' perceptions on the topics presented in the instrument.

The committee process resulted in a 32-item instrument, which appears in the Appendix. This 32-item questionnaire was next distributed to two groups of students to ensure clarity of the instrument. Feedback from students led to modifications of some items, as well as the decision to include an open-ended question for students to provide feedback on the MAKSS-TEP. Specifically, numerous students criticized the aforementioned lack of a "Neutral" option on the items, and we were interested to see whether that trend would continue if students completing the MAKSS-TEP were given the opportunity to provide feedback.

Based on the original structure of the MAKSS and the existing body of research in which iterations of the MAKSS were used, it was believed that the three scales were organized as groups of 20 items (Awareness, 1-20; Knowledge, 21-40; Skills, 41-60). After a one-semester pilot of the MAKSS-TEP, it was determined that the Awareness scale did not meet minimum standards for validity. However, a review of the updated information on the MAKSS-Form $\mathrm{T}$ (D'Andrea, Daniels, \& Noonan, 2003) revealed that factor analysis with an updated sample had resulted in different scales than what was presented in the earlier incarnation.

\section{Method}

The MAKSS-TEP was administered to students in a teacher preparation program in the rural Midwest near the end of each semester. All students completing the course focusing on multicultural education were asked to complete the MAKSSTEP; secondary education majors and elementary education majors were also asked to complete the MAKSS-TEP at the end of their student teaching semester and in an earlier methods course. As the intention is to compare students' multicultural competence at different points in their development as pre-service teachers, the same students may complete the MAKSS-TEP more than once. 
Given the relatively small enrollment of the program and the perceived need for anonymity to assure accurate responses, students were not asked to provide details about age, ethnicity, or gender. For the sake of data disaggregation, students are asked to state whether they are graduates or undergraduates, as well as whether they are studying elementary education or secondary education.

\section{Participants}

To date, 190 separate submissions of the MAKSS-TEP have been completed. Of the students completing the MAKSS, $64 \%(n=121)$ were elementary education majors, with $27 \%(n=52)$ identifying as secondary education majors. Graduate students comprised $12 \%(n=23)$ of the responses, with $68 \% \quad(n=130)$ of the responses coming from undergraduates. This sample is sufficiently representative of the composition of students in the program. There are presently just under 200 students enrolled, with a mean age of 21.5 years. The total program enrollment (including both elementary and secondary education majors) is nearly $80 \%$ female, with that percentage increasing among elementary education majors. The ethnicity of students was nearly $96 \%$ white, with another $2.5 \%$ of students identifying as multiracial. African-American, Asian, and Latinx students collectively represented about $2 \%$ of the program enrollment.

\section{Validity and Reliability of the MAKSS-TEP}

Factor analysis was conducted by selecting items on the MAKSS-TEP which matched the updated Awareness, Knowledge, and Skills subscales determined by D'Andrea, Daniels, and Noonan (2003). Five items on the MAKSS-TEP were listed among the eight items established for the Awareness scale on the MAKSSForm T, but only four items loaded at the accepted threshold of .40 or above. The range of item loadings for the Awareness scale was between .72 and .80. For the Knowledge scale of the MAKSS-TEP, eight items were included among the 13 items established by the MAKSS-Form T. Item loadings for the Knowledge scale ranged between .67 and .79. The Skills scale of the MAKSS-TEP had 10 items in common with the 20-item Skills scale of the MAKSS-Form T. Item loadings for the Skills scale ranged between .56 and .71 .

Cronbach alpha reliability coefficients were calculated for each of the relevant subscales. The four items included in the Awareness subscale produced a reliability coefficient of .77, which was comparable to the value of .73 presented by D'Andrea et al. (2003). Prior research using the MAKSS has often found the Awareness scale to have the lowest overall reliability coefficient, and this pattern held for the MAKSS-TEP. The eight items on the Knowledge subscale produced a reliability coefficient of .87 , which was nearly identical to the coefficient of .86 derived by D'Andrea et al. (2003). The 10-item Skills subscale of the MAKSSTEP produced a reliability coefficient of .81 , which differed from the .93 coefficient presented by D'Andrea et al. (2003). The Skills scale on the MAKSSTEP was the most different in both number of items and changes to content, so it would be reasonable to view the Skills subscale on the MAKSS-TEP as sufficiently reliable (i.e., a coefficient above .70) but less directly comparable to the MAKSS-Form T. 
To allow a fuller comparison to D'Andrea et al. (2003), the inter-correlation of all three subscales on the MAKSS-TEP was measured. The inter-correlation coefficient between the Awareness and Knowledge subscales for the MAKSSTEP was .75, as compared to a coefficient of .62 for the MAKSS-Form T. The inter-correlation coefficient between the Knowledge and Skills subscales of the MAKSS-TEP was found to be .62, in contrast to the .54 measured for the MAKSSForm T. The inter-correlation coefficient for the Awareness and Skills subscales of the MAKSS-TEP was .63, which differed from the .50 found for the MAKSSForm T. Overall, these tests would suggest that the three subscales adapted for the MAKSS-TEP were valid and reliable measures of distinct factors, but also were correlated sufficiently for the MAKSS-TEP to be considered a broader measure of multicultural competency.

\section{A Proposed Partial Measure of White Fragility}

After deriving and testing the three subscales of the MAKSS-TEP as described above, a total of 10 survey items existed on the MAKSS-TEP which had been deleted from the MAKSS-Form T. A total of five of those items demonstrated sufficient factor loading to be considered a valid subscale. Item loadings for this scale ranged from .47 to .69 , and included among this group was the item previously deleted from the Awareness scale.

This set of five items was also tested for reliability, in order to determine whether it could be collapsed into a new subscale. The five items produced a Cronbach's alpha of .47, which precluded this from being considered a valid or reliable subscale in its present composition. Further testing indicated that a significant positive correlation of low magnitude $(\mathrm{r}(188)=.16, \mathrm{p}<.05)$ existed between this cluster of five items and the Skills subscale of the MAKSS-TEP. The five items remaining were as follows:

1. Culture is not external but is within a person.

2. Ambiguity and stress often result from multicultural situations because people are not sure what to expect from each other.

3. In the early grades of formal schooling in the United States, the academic achievement data of such ethnic minorities as African Americans, Hispanics, and Native Americans is close to the achievement of White mainstream students.

4. Data indicates that in the early elementary school grades, girls and boys achieve about equally in mathematics and science.

5. Most of the immigrant and ethnic groups in Europe, Australia, and Canada face problems similar to those experienced by ethnic groups in the United States.

Subsequent to analyzing the five items above and participants' responses to them, a new hypothesis arose that this portion of the survey could function as an emerging or partial measure of white fragility. Although these five items did not meet statistical criteria to be treated as a valid and reliable subscale, comparing and contrasting responses on these five items with the three validated subscales of the MAKSS-TEP stands to enrich the discussion of 
findings for this study. Additionally, the question of whether this could be a basis for deliberately developing a valid and reliable measure of white fragility will be discussed further as an implication of this research.

\section{Results}

Because a sufficient number of responses were not collected for the secondary methods course, a comparison between responses for secondary capstone, elementary capstone, elementary methods, and multicultural education courses was the most responsible way to analyze data based on course enrollments. Accordingly, mean scores for each individual course will be presented and compared, and elementary methods will be compared to elementary capstone to determine if a significant change occurred over time. Data were also analyzed to explore differences between undergraduate and graduate students, as well as differences between elementary education majors and secondary education majors. When examining the data presented below, it is also important to understand that the multicultural education course is comprised of students from both majors and both degree tracks.

\section{Comparison by Course}

A general trend in scores for all subscales was that secondary education students self-rated highest for the three subscales (Awareness, Knowledge, and Skills). It needs to be noted that the sample was also the smallest for this group, and further cycles of data will reveal whether this trend persists. It is also notable that the mean score for the proposed partial measure of white fragility was also the highest among the secondary capstone students. Mean differences between secondary capstone and all other courses were found to be statistically significant $(\mathrm{p}<.05)$ for both the Knowledge and Skills subscales. The mean difference between secondary capstone was only significantly higher than the elementary methods course for the Awareness subscale.

Students completing the multicultural education course collectively self-rated higher on measures of Awareness, Knowledge, and Skills than the students in both elementary education courses. Although the mean scores were higher, no significant difference was determined between the scores for elementary methods and elementary capstone on any of the three subscales, nor for the total MAKSS-TEP. A full listing of the means and number of responses for each of the four courses (Multicultural Education, Elementary Methods, Elementary Capstone, and Secondary Capstone) appears in Table 1.

It was further notable that mean scores for the proposed partial measure of white fragility were lower after completing the multicultural education course than after completing the elementary methods course. Further, mean scores for the proposed partial measure of white fragility were also highest for the secondary capstone students. Results of a one-way ANOVA demonstrated that no significant difference existed between any of these four courses for the proposed partial measure of white fragility. 


\section{Comparison by Major}

Consistent with findings when analyzing data by course, the students majoring in secondary education had higher mean scores for the Awareness, Knowledge, and Skills subscales. The difference between elementary and secondary education majors was determined to be statistically significant $(p<.05)$ for the Knowledge subscale. The mean difference for Skills between the two groups was on the verge of statistical significance $(p=.05)$. A full summary of the means and numbers of participants in each major is presented in Table 2. This discrepancy between elementary and secondary education majors will be a topic for further scrutiny as pre-service teachers continue to complete the MAKSSTEP. Notably, secondary education majors begin direct coursework in pedagogy much later than elementary education majors, so this finding raises questions about the reasons for secondary education majors to exhibit significantly greater confidence in their multicultural knowledge and skills.

Table 1: Mean Scores for Subscales Organized by Course

\begin{tabular}{lll}
\hline & White & \\
Course & Fragility Awareness Knowledge Skills
\end{tabular}

\begin{tabular}{llllll}
\hline Multicultural & Mean & 12.58 & 12.66 & 35.50 & 29.88 \\
Education & $\mathrm{N}=$ & 50 & 50 & 42 & 43 \\
& & & & & \\
Elementary & Mean & 12.73 & 11.78 & 32.30 & 28.16 \\
Methods & $\mathrm{N}=$ & 52 & 50 & 49 & 48 \\
& & & & & \\
Elementary & Mean & 12.48 & 12.00 & 32.90 & 29.06 \\
Capstone & $\mathrm{N}=$ & 47 & 47 & 44 & 47 \\
& & & & & \\
Secondary & Mean & 13.16 & 13.41 & 39.83 & 33.09 \\
Capstone & $\mathrm{N}=$ & 12 & 12 & 12 & 11 \\
\hline
\end{tabular}

\section{Comparison by Degree}

Overall, students seeking graduate degrees exhibited higher mean scores than students pursuing undergraduate degrees. A summary of the means and numbers of participants in each degree category is presented in Table 3. Despite the difference in degree status, it should also be noted that all students represented in these data are seeking initial licensure, and are therefore largely comparable in terms of their direct experience in classroom teaching situations. 
Results of a one-way ANOVA demonstrated that mean scores for graduate students were only significantly higher for the Awareness subscale. Graduate students did also exhibit a slightly higher mean score for the proposed partial measure of white fragility, but this difference was not determined to be statistically significant.

Table 2: Mean Scores for Subscales Organized by Major

\begin{tabular}{lllll}
\hline Major & White Fragility & Awareness & Knowledge & Skills \\
\hline $\begin{array}{l}\text { Elementary Education } \\
(\mathrm{n}=121)\end{array}$ & 12.57 & 12.14 & 33.53 & 29.15 \\
$\begin{array}{l}\text { Secondary Education } \\
(\mathrm{n}=51)\end{array}$ & 12.62 & 12.54 & 35.97 & 30.47 \\
\hline
\end{tabular}

Table 3: Mean Scores for Subscales Organized by Degree Sought

\begin{tabular}{lllll}
\hline Degree Status & $\begin{array}{l}\text { White } \\
\text { Fragility }\end{array}$ & Awareness & Knowledge & Skills \\
\hline $\begin{array}{l}\text { Undergraduate Student } \\
(\mathrm{n}=130)\end{array}$ & 12.46 & 11.97 & 33.66 & 29.29 \\
$\begin{array}{l}\text { Graduate Student } \\
(\mathrm{n}=22)\end{array}$ & 13.09 & 12.86 & 34.85 & 29.47 \\
\hline
\end{tabular}

\section{Limitations}

First, it is essential to clarify that the MAKSS-TEP relies on the self-ratings of students enrolled in a teacher preparation program. This information can be valuable, and the three main subscales of the instrument have been demonstrated to meet requirements for reliability and validity. However, these data should be understood primarily as a representation of participants' own perceptions and ultimately should be part of more extensive data collection that also includes direct evidence of pre-service teachers' multicultural competence when planning and presenting instruction. As emphasized by Spanierman et al. (2011):

"Multicultural competence can (and should) be assessed through a number of methods (e.g., observations of classroom teaching, parent and/or student ratings of teachers, examination of curricula and lesson plans, and so forth). In addition to these methods, a survey instrument grounded in the extant literature that measures teachers' self-reported multicultural teaching competence would provide an efficient method of 
assessment to understand which approach works for whom under what circumstances" (p. 442).

\section{Discussion}

A concept related to white fragility is a notion of white innocence, described by DiAngelo (2011) as a sense that race and related tensions or social injustices are somehow removed from the world of whiteness which is understood to be normal and for which any disruption may be viewed as a grievous intrusion. Orozco and Diaz (2016) aligned white innocence with altruism in the larger field of education, noting that a history of questionable or harmful policies and attitudes were justified by the sense that white professionals and policymakers were making beneficial decisions on behalf of students of color. This form of altruism-as-white-innocence represented a social and educational trend that is in danger of persisting if not directly questioned and confronted in the process of developing culturally competent educators.

When examined through a lens of white fragility and altruism-as-whiteinnocence, trends on the MAKSS-TEP may take on new dimensions. Even having acknowledged the inherent limitations of participants self-rating aspects of their cultural competence, the three subscales of the MAKSS-TEP may be especially prone to inflated scores in contexts where white normativity or altruism-as-white-innocence thrive. One example of this would be that the Knowledge scale is ostensibly composed of a series of terms for which students are asked to rank their understanding. There is every reason to think that a participant would overstate their grasp of terms like culture, racism, or prejudice when not required to explain potential nuances or confront specific questions about these concepts. In other words, a participant could claim to be very familiar with racism, while ultimately holding an uncritical perspective that racism is a flaw or choice of misguided individuals which occurs separate from their white innocence and with which they are not obligated to engage.

Large portions of the Awareness and Skills subscales may be similarly at odds with the notion of altruism-as-white-innocence. For example, there is always potentially value in the self-rating or self-reflection sought by the four items on the Awareness subscale, but high ratings could just as easily be measures of unexamined whiteness as indicators of emerging cultural competency. A number of items on the Skills subscale focus on identifying bias or identifying challenges faced by students from diverse cultural backgrounds, for which it would be similarly difficult to disentangle white innocence/white fragility from legitimate skills in navigating complex social and institutional situations as a culturally responsive educator. Further, a number of items on the Skills subscale pertain to assessing the needs of groups of students based on gender, language diversity, and dis/ability status, or socioeconomic background. This portion of the MAKSS-TEP would likely be the most vulnerable to being affected by a sense of altruism rooted in whiteness (or the accompanying sense of normativity associated with being a member of a dominant group). Essentially, there is a notable difference between perceiving the assessment of students' needs as something done to them (or for their own good), rather than as something done responsively and in collaboration with students. 
In an effort to further analyze the proposed partial measure of white fragility, the five individual items were compared to each other using a Pearson correlation. Item 5, which pertained to the idea that immigrant/ethnic groups in the U.S. experienced problems similar to those in other traditionally Anglophone or white countries. Item 5 significantly correlated $(p<0.05)$ with the other four items in this proposed partial measure of white fragility, with correlation coefficients of low-to-moderate magnitude. In other words, participants who agreed that immigrant/ethnic groups faced similar problems throughout predominantly white countries were also more likely to believe that academic achievement was basically equivalent between different ethnicities and genders, that culture was a largely intrinsic phenomenon, and that multicultural situations presented inherent stress or uncertainty. This can be interpreted in a variety of ways, and is open to being read as either a defense of the status quo or as a general reluctance to engage more critically with topics of race, culture, or social justice.

For further comparison, the individual items on the proposed partial measure of white fragility were compared to the mean scores on the three subscales of the MAKSS-TEP, as well as to the total mean score which is understood to serve as a broad measure of cultural competence. No significant positive or negative relationship was found between any of the individual items and the total scale using a Pearson correlation. The only significant correlation discovered was of low magnitude $(\mathrm{r}(188)=.16, \mathrm{p}<.05)$ and existed between Item 2 (Ambiguity and stress often result from multicultural situations because people are not sure what to expect from each other) and the Knowledge subscale. Given that the Knowledge subscale ostensibly reflects confidence with terminology, one implication of the correlation with Item 2 is that participants who expressed greater confidence with terminology were recognizing that a general lack of information about multicultural topics could create anxiety or negatively impact interactions.

Despite the fact that the proposed partial measure of white fragility does not meet validation criteria to function as a scale, it did yield two significant correlations when treated as a scale. Specifically, significant correlations of low magnitude existed between the proposed partial measure of white fragility and both the Skills subscale and the total MAKSS-TEP $(\mathrm{rs}(188)=.17, \mathrm{p}<.05)$. One implication of this is that participants who are less likely to engage critically with complex social topics are also more likely to overrate their competence with skills. It is further notable that the items on the Skills subscale largely pertain to assessing the needs of different categories of students recognizing biases, or seeking out resources.

The Skills subscale and its aforementioned correlation with the proposed partial measure of white fragility could influence future research. As noted by Iverson (2012), a criticism of how skills have often been perceived in the context of multicultural education has been "well-intentioned by naïve attempts to develop behaviors that are culturally sensitive," but which ultimately fail to "address the 
application of one's understanding" (p. 69). The phrasings and descriptions of the items on the Skills subscale of the MAKSS-TEP were arguably passive in nature. It is therefore possible that these data captured a reflection that passive skills are closer to disengagement or inaction than to socially conscious action. One implication is that a connection exists between this more passive presentation of multicultural skills and the inertia that is characteristic of white fragility. Future studies might also benefit from developing and validating a Skills subscale that describes direct and specific actions, rather than hinging largely on participants' readiness to address the needs of different groups within the student population.

DiAngelo (2012) posited that many white people have been socially conditioned to avoid discussions of race, using what is termed white silence to deny the reality that "there is no race-neutral space" (p. 4). This particular strand of critical whiteness may serve as a relevant connection to the responses to the final, open-ended question on the MAKSS-TEP. At the end of the questionnaire, participants were given the opportunity to provide questions or feedback. To date, around $25 \%$ of participants have responded to this question, and the majority of responses have expressed some frustration over the language of the survey or indicated that there should be a "Neutral" option on the scale. Examples of participant responses included the following:

"I was in between agree and disagree on some questions. It was difficult to have no in between option."

"For the first group of questions I think there needs to be an option between agree and disagree."

"Yes, it would help greatly if there was a neutral option if lacking an answer one way or the other."

"It might be helpful if there was a neutral option that we could choose."

"I would add an "I'm not sure" option or "I don't know" option to some of the questions."

While it is plausible to suggest that participants expressed these sentiments partly because of the ubiquity of survey instruments that do provide a neutral option, the nature of this particular instrument and composition of the sample merit further discussion. As noted by DiAngelo (2012), "Not contributing one's perspectives serves to ensure that those perspectives cannot be expanded" and "The role of silent whites is critical to protecting whiteness, for white dominance depends, in part, on the silence of other whites" (p. 5). The responses presented above, along with the comments from the original test groups of students about needing a neutral option, could represent a subtle expression of hidden rules of whiteness. Specifically, white silence is understood to be not only an acceptable norm, but also potentially a tool which can be freely leveraged to avoid engaging in difficult discussions on topics of race or social inequity. 


\section{Directions for Future Research}

As noted above, the process of collecting and analyzing data using the MAKSSTEP raised the question of whether five items originally believed to be part of the Awareness subscale were in fact a subtle, partial measure of white fragility. A likely direction for future research will be the investigation of whether a valid and reliable measure of white fragility can be developed, using these five items as a starting point. This endeavor, and really any future projects growing from this initial testing of the MAKSS-TEP, will depend upon being able to gather data from a larger sample which would ideally include participants from different cultural and linguistic backgrounds, and from different regions of the United States.

Another possibility for future research would be to add open-ended questions to several of the more ambiguous MAKSS-TEP items. For example, responses to Item 5 (School problems vary with the culture of the student) have been difficult to interpret, as there is currently no way to know whether participants who agree with this statement are doing so because they believe students from traditionally marginalized groups have typically had more difficult interactions with the U.S. public education system. Simply adding a follow up question to the survey asking participants to explain their responses to Item 5 would yield richer information to interpret in the future.

\section{Conclusion}

The goal of preparing culturally competent K-12 educators has been an ongoing priority, and self-rating instruments can be used in concert with other sources of information to ensure that teacher preparation programs fulfill their obligations to this process. Given the increasingly volatile climate of the contemporary United States and the rich theoretical framework that exists for critically examining whiteness, there is a heightened need to confront topics like white fragility in honest and compassionate ways. An instrument like the MAKSSTEP may very well serve as at least a partial measure of white fragility, but it is only valuable to the extent that it sparks responsible discussion and prompts meaningful changes to programs and other experiences for students in teacher preparation programs. The ultimate intent is not to capture or vilify, but instead to give future educators a starting point for growth. As DiAngelo (2018) concluded, confronting issues of whiteness and dismantling racist structures can be initially difficult, but should lead to a lifelong process of thinking and acting critically. In the end, confronting white fragility is intended to be liberating, and it undoubtedly can be, both for students in teacher preparation programs and for the K-12 students they will eventually teach.

Many thanks to Dr. Denise Dallmer of Indiana University East for her valuable contributions to the development of this study. 


\section{References}

Banks, J. A. (1995). Multicultural Education: Historical Development, Dimensions, and Practice. In J. A. Banks \& C. M. Banks (Eds.). Handbook of Research on Multicultural Education (pp. 3-24). New York: Macmillan. doi.: 10.2307/1167339

Center for the Study of Hate and Extremism. (2018). Report to the Nation: Hate Crimes Rise in U.S. Cities and Counties in Time of Division and Foreign Interference. Retrieved from https://csbs.csusb.edu/sites/csusb_csbs/files/2018\%20Hate\%20Final\%20Repor t\%205-14.pdf

D'Andrea, M., \& Daniels, J. (1999). Exploring the psychology of white racism through naturalistic inquiry. Journal of Counseling and Development, 77, 93-101. doi.: 10.1002/j.1556-6676.1999.tb02426.x

D'Andrea, M., Daniels, J., \& Heck, R. (1991). Evaluating the impact of multicultural counseling training. Journal of Counseling \& Development, 70, 143-150. doi: 10.1002/j.1556-6676.1991.tb01576.x

D'Andrea, M., Daniels, J., \& Noonan, M. J. (2003). New developments in the assessment of multicultural competence: The Multicultural Awareness-Knowledge-Skills Survey-Teachers Form. In D. B. Pope-Davis, H. L. K. Coleman, W. M. Liu, \& R. L. Toporek (Eds.), Handbook of competencies in counseling and psychology (p. 154167). Thousand Oaks, CA: Sage Publications, Inc. doi: $10.4135 / 9781452231693 . n 10$

DiAngelo, R. (2018). White fragility. Boston, MA: Beacon Press.

DiAngelo, R. (2012). Nothing to add: A challenge to white silence in racial discussions. Understanding \& Dismantling Privilege, 2(1), 2-17.

DiAngelo, R. (2011). White fragility. International Journal of Critical Pedagogy, 3(3), 54-70.

Frankenberg, E. (2008). The segregation of American teachers. Education Policy Analysis Archives, 17(1), 1-44. doi: 10.14507/epaa.v17n1.2009

Hall, J. C., \& Theriot, M. T. (2016). Developing multicultural awareness, knowledge, and skills: Diversity training makes a difference? Multicultural Perspectives, 18(1), 3541. doi: 10.1080/15210960.2016.1125742

Iverson, S. V. (2012). Multicultural competence for doing social justice: Expanding our awareness, knowledge, and skills. Journal of Critical Thoughts and Praxis, 1(1), 6287. doi: $10.31274 /$ jctp-180810-14

Jones, J., \& Walker, S. L. (2016). The development of the multicultural awareness, knowledge, and skills survey: An instrument for assessing the cultural competency of pre-service teachers. Diversity, Social Justice, and the Educational Leader, 1(2), 40-54.

Ladson-Billings, G. (1994). The dreamkeepers: Successful teaching for African-American students. San Francisco: Jossey-Bass. doi:10.3726/978-1-4539-1735-0/39

National Center for Educational Statistics. (2017). Racial/Ethnic Enrollment in Public Schools. Retrieved from https://nces.ed.gov/programs/coe/pdf/coe_cge.pdf

National Center for Education Statistics [NCES]. (2015). Characteristics of postsecondary faculty. In The Condition of Education 2015 (pp. 226-229). Washington, DC: Department of Education. Retrieved from https://nces.ed.gov/pubs2015/2015144.pdf

Orozco, R., \& Diaz, J. J. (2016). "Suited to their needs": White innocence as a vestige of segregation. Multicultural Perspectives, 18(3), 127-133.

doi: 10.1080/15210960.2016.1185610 
Prieto, L. R. (2012). Initial factor analysis and cross-validation of the Multicultural Teaching Competencies Inventory. Journal of Diversity in Higher Education, 5(1), 50-62. doi: 10.1037/a0026199

Sensoy, O., \& DiAngelo, R. (2017). “We are all for diversity, but...” How faculty hiring committees reproduce whiteness and practical suggestions for how they can change. Harvard Educational Review, 87(4), 557-580. doi: 10.17763/1943-504587.4.557

Southern Poverty Law Center. (2019, Spring). Intelligence Report. Retrieved from https://www.splcenter.org/sites/default/files/intelligence_report_166.pdf

Spanierman, L. B., Oh, E., Heppner, R. P., Neville, H. A., Mobley, M., Wright, C. V., Dillon, F. R., \& Navarro, R. (2011). The multicultural teaching scale: Development and initial validation. Urban Education, 46(3), 440-464. doi: $10.1177 / 0042085910377442$

Warring, D. F. (2005). Assessing student multicultural attitudes, knowledge, and skills in teacher education. Journal of Thought, 40(3), 107-119. 


\section{Appendix}

A complete list of the items included on the MAKSS-TEP instrument, adapted from

D'Andrea, Daniels, and Noonan (2003).

1. Culture is not external but is within a person.

2. Becoming more informed about specific cultures can also lead to stereotyping of members of those cultural groups.

3. The teaching profession has failed to meet the needs of ethnic minorities.

4. Ambiguity and stress often result from multicultural situations because people are not sure what to expect from each other.

5. School problems vary with the culture of the student.

6. There are some basic teaching skills that are applicable to create successful outcomes regardless of the student's cultural background.

7. In the early grades of formal schooling in the United States, the academic achievement data of such ethnic minorities as African Americans, Hispanics, and Native Americans is close to the achievement of White mainstream students.

8. Data indicates that in the early elementary school grades, girls and boys achieve about equally in mathematics and science.

9. Most of the immigrant and ethnic groups in Europe, Australia, and Canada face problems similar to those experienced by ethnic groups in the United States.

10. The concept of "integration" has implicit bias in favor of the dominant culture.

At the present time, how would you rate your own understanding of the following terms: (Very Limited -Limited - Good -Very Good)

11. Culture

12. Ethnicity

13. Racism

14. Mainstreaming

15. Prejudice

16. Ethnocentrism

17. Pluralism

18. Transcultural

19. At this point in your life, how would you rate your understanding of the impact of the way you think and act when interacting with students of different cultural backgrounds?

20. In general, how would you rate your level of awareness regarding different cultural institutions and systems?

21. At this time in your life, how would you rate yourself in terms of understanding how your cultural background has influenced the way you think and act?

22. At the present time, how would you generally rate yourself in terms of being able to accurately compare your own cultural perspective with that of a person from another culture? 
23. In general, how would you rate yourself in terms of being able to effectively deal with biases, discrimination, and prejudices directed at you by a student in a school?

24. How well would you rate your ability to identify culturally biased assumptions?

25. In general, how would you rate your ability to articulate a student's problem who comes from a cultural group different from your own?

26. How would you rate your ability to effectively secure information and resources to better serve culturally different students?

27. How would you rate your ability to assess the needs of male students?

28. How would you rate your ability to assess the needs of female students?

29. How would you rate your ability to assess the needs of transgender students?

30. How would you rate your ability to assess the needs of students with disabilities?

31. How would you rate your ability to assess the needs of English Language Learners?

32. How well would you rate your ability to assess the needs of persons who come from very poor socioeconomic backgrounds? 